\title{
Anticoagulation Treatment in Cancer-Associated Venous Thromboembolism: Assessment of Patient Preferences Using a Discrete Choice Experiment (COSIMO Study)
}

Nils Picker ${ }^{1}$ Agnes Y. Lee ${ }^{2,3}$ Alexander T. Cohen ${ }^{4}$ Anthony Maraveyas ${ }^{5}$ Jan Beyer-Westendorf ${ }^{6,7}$ Lorenzo G. Mantovani ${ }^{8,9}$ Khaled Abdelgawwad ${ }^{10}$ Samuel Fatoba ${ }^{10}$ Inga-Marion Thate-Waschke ${ }^{11}$ Miriam Bach ${ }^{10}$ Thomas Wilke ${ }^{1}$

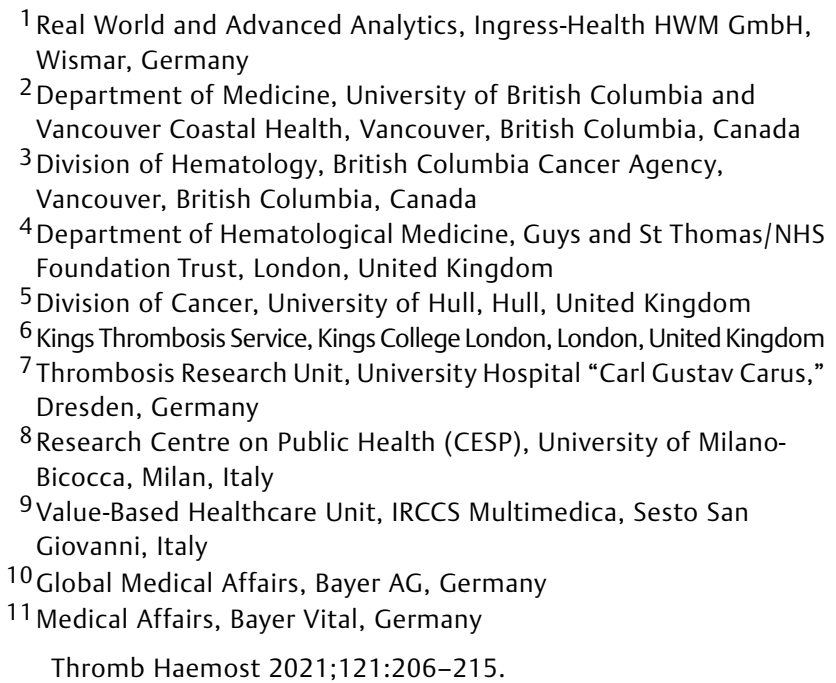

Address for correspondence Nils Picker, MSc, Ingress-Health HWM GmbH, Alter Holzhafen 19, 23966 Wismar, Germany (e-mail: nils.picker@ingress-health.com).

\begin{abstract}
Keywords

- anticoagulation

- patient preferences

- treatment-related decision making

- discrete choice experiment

- cancer-associated venous thromboembolism
\end{abstract}

Introduction Clinical guidelines recommend anticoagulation therapy for the treatment of cancer-associated venous thromboembolism (VTE), but little is known about preferences. Therefore, the objective of this discrete choice experiment (DCE) was to elucidate patient preferences regarding anticoagulation convenience attributes.

Methods Adult patients with cancer-associated VTE who switched to direct oral anticoagulants were included in a single-arm study (COSIMO). Patients were asked to decide between hypothetical treatment options based on a combination of the following attributes: route of administration (injection/tablet), frequency of intake (once/twice daily), need for regular controls of the international normalized ratio (INR) at least every 3 to 4 weeks (yes/no), interactions with food/alcohol (yes/no), and distance to treating physician ( $1 \mathrm{vs.} 20 \mathrm{~km}$ ) as an additional neutral attribute. DCE data were collected by structured telephone interviews and analyzed based on a conditional logit regression.

Results Overall, 163 patients (mean age 63.7 years, 49.1\% female) were included. They strongly preferred oral administration compared with self-injections (importance received

March 23, 2020

accepted after revision

June 25,2020

published online

September 2, 2020
DOI https://doi.org/

$10.1055 / \mathrm{s}-0040-1714739$. ISSN 0340-6245.

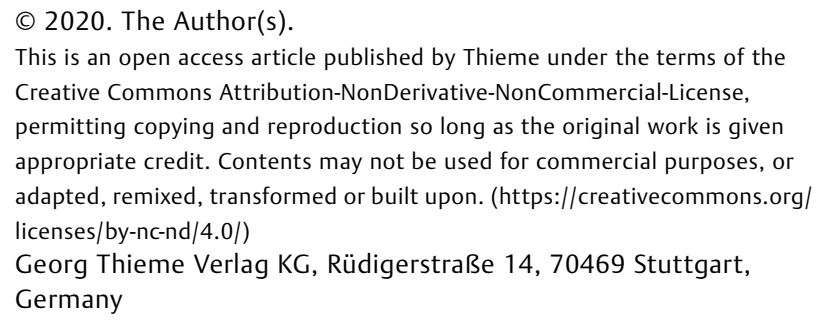


of this attribute for overall treatment decisions: 73.8\%), and a treatment without dietary restrictions (11.8\%). Even if these attributes were less important $(7.2 \%$ and $6.5 \%$, respectively), patients indicated a preference for a shorter distance to the treating physician and once-daily dosing compared with twice-daily intake. "Need for regular controls of INR at least every 3 to 4 weeks" showed no significant impact on the treatment decision $(0.7 \%)$.

Conclusion This study showed that treatment-related decision making in cancerassociated VTE, assuming comparable effectiveness and safety of anticoagulant treatments, is predominantly driven by "route of administration," with patients strongly preferring oral administration.

\section{Introduction}

Venous thromboembolism (VTE, which includes deep vein thrombosis [DVT] and pulmonary embolism [PE]) is a common complication in patients with cancer. Previous guidelines have recommended low-molecular-weight heparin (LMWH) over vitamin Kantagonists (VKAs) for the treatment of these patients; the direct oral anticoagulants (DOACs) have only recently been included in these recommendations. ${ }^{1}$ LMWH and VKAs have several drawbacks that can increase the burden of disease in patients. DOACs, such as rivaroxaban, can ameliorate some of these burdens and improve health-related quality of life $(\mathrm{QoL})^{2,3}$

Health care providers base treatment decisions mainly on effectiveness and safety. Although anticoagulation therapy has been shown to be reasonably effective and safe in patients with cancer-associated VTE, there is a shortage of information around the anticoagulant preferences of these patients regarding type, pharmacological profile, benefits, and drawbacks. Such preferences could be an important driver of treatment adherence, persistence, and clinical outcomes because patients with cancer-associated VTE often require long-term therapy. ${ }^{4}$ Moreover, prescribing treatments that patients prefer may also generally strengthen the relationship between the physician and the patient. Thus, guidelines recommend incorporating patient preferences in the decision-making process. ${ }^{5}$

The primary objective of the COSIMO (Cancer-associated thrOmboSIs-patient-reported outcoMes with rivarOxaban) study was to provide real-world information on treatment satisfaction in patients with active cancer, who switch from traditional anticoagulants (LMWH or VKA) to rivaroxaban for the treatment of VTE. Therefore, information on conveniencerelated patient preferences in anticoagulation treatment was collected as secondary outcome to gain a better understanding of what influences treatment satisfaction.

To the knowledge of the authors, only a few studies have addressed preferences of patients with cancer-associated VTE in terms of anticoagulation treatment and most of these predated DOAC therapy in this setting. ${ }^{6,7}$ Thus, more data on anticoagulant preferences of patients with cancer-associated VTE are needed. The aim of our research was to evaluate patient preferences regarding convenience attributes that characterize different anticoagulation options for patients with cancer-associated VTE in multiple countries. Switching in anticoagulation treatment is common in cancer patients to balance their changing needs or stages of disease. This will most likely affect patient's convenience-related preferences. Consequently, this study evaluates only patients switching from conventional anticoagulation to rivaroxaban.

\section{Methods}

\section{Study Design}

We conducted a discrete choice experiment (DCE) within the COSIMO study. COSIMO was a multinational, prospective, noninterventional, multicenter, single-arm cohort study in patients with active cancer, who changed from traditional anticoagulants (LMWH, fondaparinux/VKA) to rivaroxaban for the treatment or secondary prevention of cancer-associated VTE. ${ }^{8}$ The primary outcome in COSIMO was treatment satisfaction, which was evaluated using the Anti-Clot Treatment Scale (ACTS) based on changes in the subscale for the ACTS Burdens score at week 4 compared with baseline. The DCE was a prespecified analysis within COSIMO to examine how study participants evaluate the characteristic profile of different anticoagulants. Structural telephone interviews between week 4 and week 12 after study enrollment of each patient (start of DOAC therapy) were planned. The cross-sectional DCE survey was conducted in patients recruited from Australia, Belgium, Canada, Denmark, France, Germany, Italy, Netherlands, Spain, and the United Kingdom. A complete list of investigators/study sites contributing to patient inclusion is available as supplementary material (-Supplementary Table S1, available in the online version).

\section{Patient Sample}

The general study inclusion criteria for the COSIMO study were: age of at least 18 years; active cancer other than fully treated basal-cell or squamous-cell carcinoma of the skin (active cancer defined as the diagnosis or treatment of cancer in the previous 6 months or recurrent or metastatic cancer); previously treated with traditional anticoagulants (LMWH, fondaparinux/VKA) for the treatment of DVT and/or PE (index venous thromboembolic event) for at least 4 weeks; change of anticoagulant to DOAC (rivaroxaban); Eastern 
Cooperative Oncology Group performance status score of 0 , 1 , or 2 ; and available for follow-up with a life expectancy $>6$ months. Patients who developed an index venous thromboembolic event despite anticoagulant therapy or were treated with anticoagulants other than standard of care were excluded. Patients participating in an investigational program with interventions outside of routine clinical practice with the exception of oncology investigational trials were also excluded. In addition, patients participating in the DCE survey had to meet the following criteria: ability to participate in a DCE survey in one of the predefined languages (Danish, Dutch, English, French, German, Italian, or Spanish) and willingness to be interviewed over telephone as confirmed by a separate informed consent.

\section{Discrete Choice Experiment Design}

The conduct of a DCE is a widely accepted methodologic approach for patient preference elucidation in health care. Choice experiments examine preferences using pairwise comparisons of holistic hypothetical alternatives instead of ranking or assessing single features only. ${ }^{9,10}$ Each alternative is described by predefined attributes that vary across the different alternatives. The variation across the alternatives in the choice sets is achieved by assigning different levels to the attributes. The basic assumption of a DCE is that rational individuals will always choose the alternative with the higher level of expected utility. In this way, the degree to which each attribute (treatment characteristic) influences the choice of the patient can be examined. ${ }^{11,12}$

Potential convenience attributes that characterize the known alternatives for anticoagulation treatment of patients with cancer-associated VTE (LMWH, VKA, and DOAC) were developed based on a literature review, expert group discussions, and qualitative interviews with seven patients with cancer-associated VTE, to guide the development of the questionnaire for the quantitative DCE survey. At the time the DCE was designed, results from randomized controlled trials that directly compared the efficacy and safety of LMWH, VKA, and DOAC were not available. Therefore, it was predefined that no attributes describing the different efficacy or safety of these treatments were included in the experiment, and so, an assumption of similar efficacy and safety formed the basis of our DCE, which was then focused on the preference measurement of convenience attributes only.

In the qualitative interviews, patients were invited to openly discuss and rank the different attributes presented in terms of their importance. Furthermore, after each interview, patients were asked whether there were further relevant and important attributes describing their anticoagulation treatment.

Based on the attribute ratings with the highest importance, the following treatment attributes were selected for the final DCE design (- Table 1): route of administration (selfinjection or tablet), frequency of intake (once vs. twice daily), need for regular controls of international normalized ratio (INR) at least every 3 to 4 weeks (yes/no), and interactions with food/alcohol (yes/no). In addition, a comparator attribute defined as the distance to the treating physician was included to express the marginal valuations of the treatment
Table 1 Attributes and levels used in the DCE

\begin{tabular}{|l|l|l|}
\hline Attribute & Level $\mathbf{1}$ & Level 2 \\
\hline Route of administration & Oral tablet & Self-injection \\
\hline Frequency of intake & Once daily & Twice daily \\
\hline $\begin{array}{l}\text { Need for regular controls } \\
\text { of INR at least every } \\
\text { 3-4 weeks }\end{array}$ & Yes & No \\
\hline $\begin{array}{l}\text { Restrictions regarding eating } \\
\text { habits/consumption of alcohol }\end{array}$ & Yes & No \\
\hline Distance to treating physician & $1 \mathrm{~km}$ & $20 \mathrm{~km}$ \\
\hline
\end{tabular}

Abbreviations: DCE, discrete choice experiment; INR, international normalized ratio; VTE, venous thromboembolism.

Note: This table presents the selected treatment convenience attributes and their respective levels, which were derived from expert panel discussions and qualitative in-depth interviews with cancer-associated VTE patients.

attributes in terms of an easily understood unit (distance in kilometer $[\mathrm{km}])$.

A fractional factorial design was generated using IBM SPSS Statistic software (version 20). ${ }^{13}$ Based on an orthogonal main-effects design, a set of nine different choice sets, each with two alternative treatment options, was derived. A 10th choice set was integrated to assess consistency of response behavior. This test set duplicated a previous decision situation with interchanged hypothetical treatment options $A$ and B. All choice sets were graphically depicted to facilitate comprehension of the different attributes and their levels (see - Fig. 1 for an example choice set used in the study).

\section{Data Collection}

The DCE telephone interviews were conducted by trained interviewers and supported by an electronic interview guide. Interviews took place 4 to 12 weeks after the change from traditional anticoagulants to DOAC (study inclusion date). Several days before an interview started, patients were provided with choice cards (sent by post), which graphically visualized the treatment choices addressed in this study. The presence of the DCE cards was a prerequisite for all patient interviews.

Treatment-related data as well as demographic and clinical patient characteristics were collected by use of an electronic data capture system from medical records and during visits that took place in routine practice.

\section{Statistical Analysis}

Baseline patient characteristics were analyzed using descriptive statistics. Categorical data were reported as proportions and continuous data as means with standard deviation (SD). Patients with inconsistent DCE data were excluded from the analyses. Inconsistency was assessed based on responses in a test set displaying an identical choice set with a reversed order of choice alternatives.

The DCE dataset was analyzed using a conditional logit regression model that included all attributes (anticoagulation treatment characteristics) as independent variables. The conditional logit relates to the probability of choice among the alternatives (choice sets) to the characteristics of the 


\section{Option 1A}

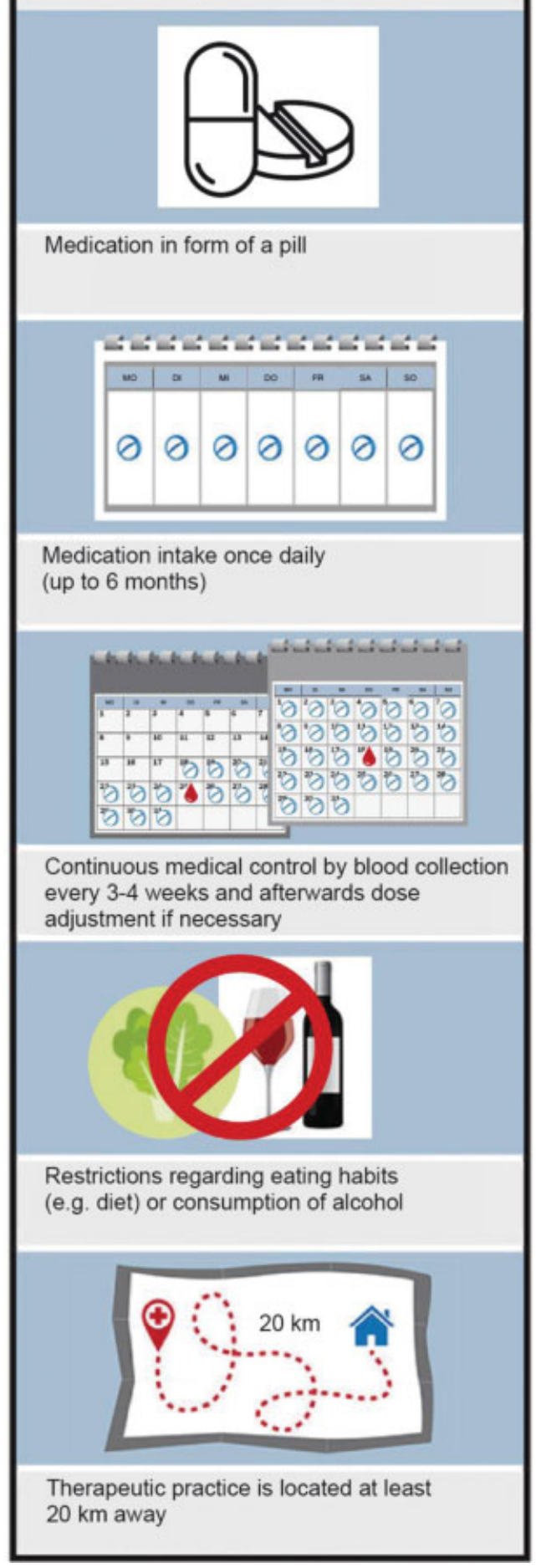

\section{Option 1B}

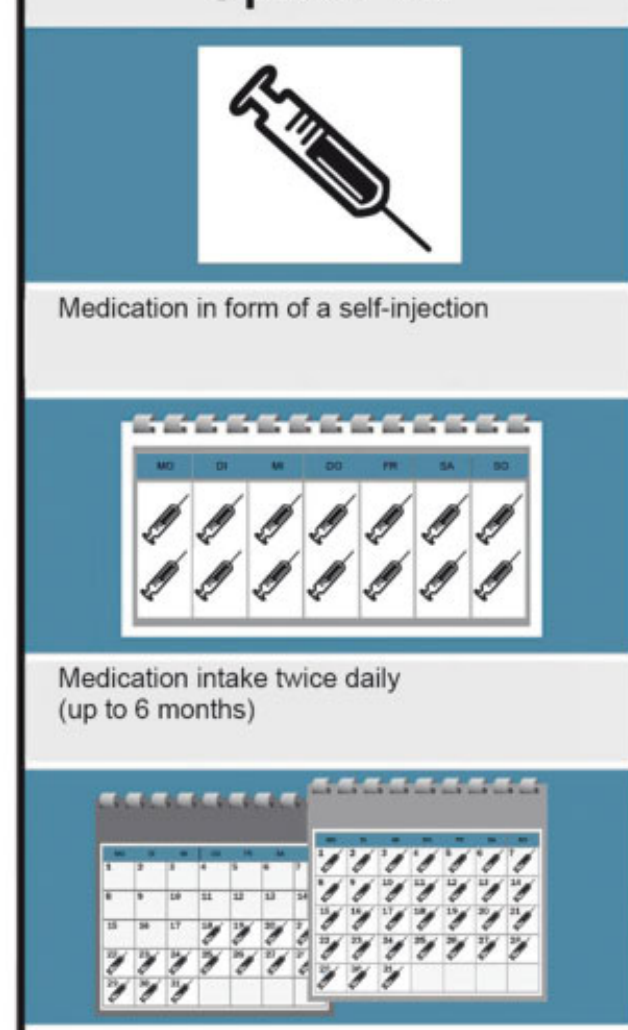

No need for continuous medical control by blood collection every $3-4$ weeks and subsequent potential dose adjustments

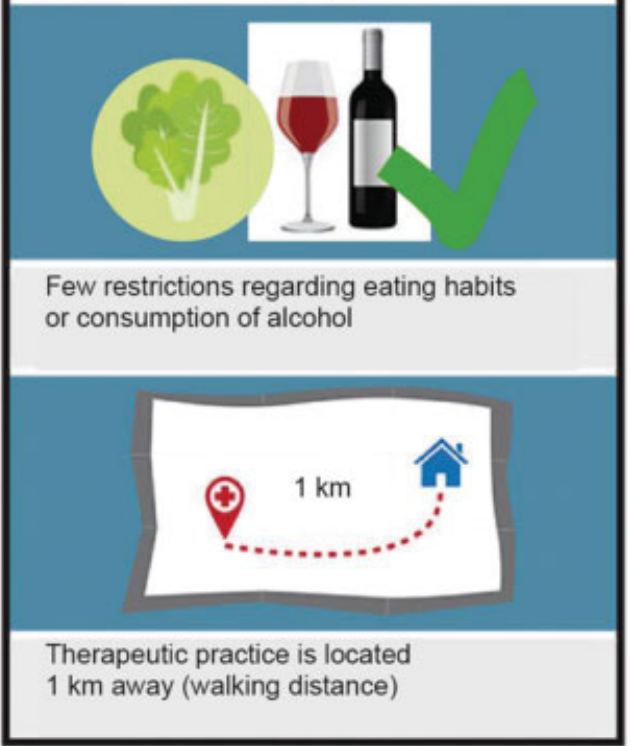

Fig. 1 Example of a visualized DCE set. The DCE card shows two hypothetical DOAC alternatives derived from a combination of the defined attributes and their respective levels. Patients were required to decide between option A or B (no opt-out). DCE, discrete choice experiment; DOAC, direct oral anticoagulant.

attribute levels defining those alternatives. ${ }^{11}$ The relative importance of each attribute for the overall decision was calculated based on the absolute distance between the minimum and maximum coefficients of the level of each attribute. The relation of the coefficient distance of each attribute to the sum of the absolute coefficient distances of all attributes can be interpreted as the relative importance. These were estimated for the overall sample as well as for prespecified subgroups. Subsequently, we calculate the marginal rate of substitutions as the distance a patient is willing to travel as the quotient between the coefficient for each convenience-related attribute $\left(\beta_{i}\right)$ and the coefficient for the 
neutral comparator "distance to the treating physician" $\left(\beta_{k}\right)$ by applying the following formula:

$$
\left|\frac{\beta_{i}}{\beta_{k}}\right|
$$

To control for preference heterogeneity in the mean, we have interacted the attributes with patients' individual characteristics. We considered the following subgroup covariates for our statistical analyses: age coded $=1$ for the oldest $50 \%$ of the sample ( $>64$ years old, else zero), gender, burden of treatments (measured by ACTS, coded $=1$ for ACTS above median [>55]), alcohol use (vs. abstinent patients), and continent of origin (European vs. Non-European). All analyses were done with Stata/MP 14 and Microsoft Excel 2016.

\section{Ethical and Regulatory Aspects}

All procedures for the survey were conducted in accordance with the legal and ethical guidelines as laid out by the European Medicines Agency, Food and Drug Administration, and applicable local laws and regulations. ${ }^{14}$ Before documentation of any data, informed consent was obtained from the patient in writing. This study was an observational study, where rivaroxaban was prescribed in the customary manner in accordance with the terms of the marketing authorization. There was no assignment of a patient to a particular therapeutic strategy. The treatment decision fell within current medical practice and prescription of the medicines was clearly separated from the decision to include the patient in the study. No additional diagnostic or monitoring processes were required for inclusion or during the study.

\section{Results}

\section{Baseline Characteristics}

A total of 181 patient interviews were completed within this study. Of these, 13 patients were excluded due to inconsistent responses. Another five patients withdrew their informed consent after interview completion, resulting in a DCE population of 163 patients (Australia: 3 [1.8\%], Belgium: 6 [3.7\%], Canada: 41 [25.2\%], Denmark: 11 [6.8\%], France: 12 [7.4\%], Germany: 15 [9.2\%], Italy: 15 [9.2\%], Netherlands: 10 [6.1\%], Spain: 15 [9.2\%], and the UK: 35 [21.5\%]). The mean age was 63.7 (SD: 10.7) years and 80 patients (49.1\%) were females (-Table 2). On average, patients had been diagnosed with cancer 22.4 months ago (SD: $33.8, n: 143$ ), while the mean time since their first VTE was 6.5 months ago (SD: $5.9 ; n$ : 159). With respect to status of cancer when changing to DOAC, $18.4 \%$ of patients were in remission (complete $10.4 \%$; partial: $8.0 \%$ ), $29.4 \%$ had stable disease, and $17.8 \%$ progressed or relapsed from recurrent disease. For the remaining patients, cancer states were unknown or could not be evaluated.

\section{Discrete Choice Experiment Results}

The expected utility associated with the different attribute levels and the relative importance of each attribute for patients' decision making were described by a conditional logit model (-Fig. 2). Oral administration as a tablet was
Table 2 Patient characteristics

\begin{tabular}{|c|c|c|}
\hline Variable & $\begin{array}{l}\text { COSIMO } \\
\text { safety } \\
\text { analysis set }\end{array}$ & $\begin{array}{l}\text { DCE } \\
\text { participants }\end{array}$ \\
\hline$N$ & 505 & 163 \\
\hline Age in years, mean (SD) & $64.0(11.7)$ & $63.7(10.7)$ \\
\hline $\begin{array}{l}\text { ACTS burden score at } \\
\text { week } 4 \text {, mean (SD) }\end{array}$ & $51.8(7.3)$ & $52.0(7.2)$ \\
\hline Female gender, $n(\%)$ & $280(55.4)$ & $80(49.1)$ \\
\hline Married, $n(\%)$ & $348(68.9)$ & $119(73.0)$ \\
\hline \multicolumn{3}{|l|}{ BMI, mean (SD) } \\
\hline Male & $26.6(4.4)$ & $27.6(5.1)$ \\
\hline Female & $27.4(6.3)$ & $27.7(7.6)$ \\
\hline \multicolumn{3}{|l|}{ Race, $n(\%)$} \\
\hline Asian & $12(2.4)$ & $5(3.1)$ \\
\hline Black & $6(1.2)$ & $2(1.2)$ \\
\hline Caucasian & $438(86.7)$ & $136(83.4)$ \\
\hline \multicolumn{3}{|c|}{ Currently drinking alcohol, $n$ (\%) } \\
\hline Yes & $242(47.9)$ & $93(57.1)$ \\
\hline No & $214(42.4)$ & $52(31.9)$ \\
\hline Unknown & $49(9.7)$ & $18(11.0)$ \\
\hline \multicolumn{3}{|l|}{ Smoking status, $n(\%)$} \\
\hline Current smoker & $37(7.3)$ & $11(6.8)$ \\
\hline Former smoker & $189(37.4)$ & $64(39.3)$ \\
\hline Never smoked & $266(52.7)$ & $83(50.9)$ \\
\hline \multicolumn{3}{|l|}{ ECOG PS, $n(\%)$} \\
\hline 0 & $162(32.1)$ & $54(33.1)$ \\
\hline 1 & $276(54.7)$ & $94(57.7)$ \\
\hline 2 & $63(12.5)$ & $13(8.0)$ \\
\hline $\begin{array}{l}\text { Time since cancer } \\
\text { diagnosis to DCE } \\
\text { interview date in } \\
\text { months, mean (SD) }\end{array}$ & $\mathrm{N} / \mathrm{A}$ & $22.4(33.8)$ \\
\hline $\begin{array}{l}\text { Less than } 3 \text { months } \\
\text { before the DCE, } n(\%)\end{array}$ & - & $5(3.0)$ \\
\hline $\begin{array}{l}\text { More than 3-6 months } \\
\text { before the DCE, } n(\%)\end{array}$ & - & $32(19.6)$ \\
\hline $\begin{array}{l}\text { More than } 6-12 \text { months } \\
\text { before the DCE, } n(\%)\end{array}$ & - & $45(27.6)$ \\
\hline $\begin{array}{l}\text { More than } 1 \text { year before } \\
\text { the DCE, } \mathrm{n}(\%)\end{array}$ & - & $81(49.7)$ \\
\hline $\begin{array}{l}\text { Time since last VTE event } \\
\text { in months, mean (SD) }\end{array}$ & $\mathrm{N} / \mathrm{A}$ & $6.5(5.9)$ \\
\hline \multicolumn{3}{|c|}{ Status of cancer response at study entry, $n(\%)$} \\
\hline Complete remission & $47(9.3)$ & $17(10.4)$ \\
\hline Partial remission & $38(7.5)$ & $13(8.0)$ \\
\hline Stable disease & $146(28.9)$ & $48(29.5)$ \\
\hline $\begin{array}{l}\text { Relapsed/progressive } \\
\text { disease }\end{array}$ & $89(17.6)$ & $29(17.8)$ \\
\hline Unknown/not evaluable & $185(36.6)$ & $56(34.4)$ \\
\hline \multicolumn{3}{|l|}{ Type of VTE, $n$ (\%) } \\
\hline
\end{tabular}


Table 2 (Continued)

\begin{tabular}{|c|l|l|}
\hline Variable & $\begin{array}{l}\text { COSIMO } \\
\text { safety } \\
\text { analysis set }\end{array}$ & $\begin{array}{l}\text { DCE } \\
\text { participants }\end{array}$ \\
\hline Catheter-associated DVT & $38(7.5)$ & $13(8.0)$ \\
\hline DVT only & $229(45.3)$ & $73(44.8)$ \\
\hline PE only & $188(37.2)$ & $66(40.5)$ \\
\hline DVT + PE only & $49(9.7)$ & $11(6.7)$ \\
\hline Type of cancer, $n(\%)$ & \multicolumn{3}{|l}{} \\
\hline Hematological malignancy & $56(11.1)$ & $18(11.0)$ \\
\hline Solid tumor & $449(88.9)$ & $145(89.0)$ \\
\hline
\end{tabular}

Abbreviations: ACTS, Anti-Clot Treatment Scale; BMI, body mass index; DCE, discrete choice experiment; DVT, deep vein thrombosis; ECOG PS, Eastern Cooperative Oncology Group performance status; PE, pulmonary embolism; SD, standard deviation; VTE, venous thromboembolism.

Note: This table presents the baseline characteristics for patients included in the DCE survey.

strongly preferred compared with self-injections (utility: 2.39 ; $p<0.001$ ). Patients also expressed a positive preference for independent consumption of food/alcohol with no interaction with medication (utility: $0.38 ; p<0.001$ ) and a once-daily dosing frequency over a twice-daily frequency (utility: 0.21 ; $p<0.05$ ). Conversely, patients did not put much emphasis on "need for regular controls of INR at least every 3 to 4 weeks," so that no such need was associated with an additional utility. In summary, route of administration was the most important attribute (73.8\% impact on the overall decision of patients), followed by restrictions due to interactions with food/alcohol
(11.8\%), distance to treating physician (7.2\%), and dosing frequency (6.5\%). The least important attribute was the need for regular controls of INR at least every 3 to 4 weeks (0.6\%). In addition, patients expressed a positive utility associated with a shorter distance to the treating physician $(1 \mathrm{~km})$ compared with $20 \mathrm{~km}$ (utility: $0.24 ; p<0.001$ ). By using this attribute as a neutral comparator, marginal valuations of the treatment convenience attributes were expressed as the distance a surveyed patient would be willing to travel to receive a positively assessed attribute level compared with a less preferred one ( - Fig. 3). Based on these data, patients would be willing to travel an additional $242 \mathrm{~km}$ to their treating physician to receive an anticoagulant that combines all the preferred characteristics (once-daily dosing, oral intake as a tablet, medication-independent food/alcohol consumption, and no need for regular controls of INR at least every 3-4 weeks), compared with an anticoagulant that combines all the characteristics that were not preferred. For a treatment option that provides at least an oral administration (instead of selfinjected medication), patients were willing to accept a longer distance of $192 \mathrm{~km}$. The equivalent distances for receiving a once-daily regimen and medication with less restrictions regarding food/alcohol consumption were 17 and $31 \mathrm{~km}$, respectively.

\section{Subgroup Analyses}

Our findings were generally consistent across all subgroups of patients, with oral administration strongly preferred over self-injections. While still ranking oral administration as the most important attribute, elderly patients and women seemed to put higher relative value on having a shorter distance to their treating physician compared with the overall study population (- Fig. 4). Men and younger patients (age below median: 65 years), on the other hand, revealed a

- Utilities associated with different attribute levels $=$ Relative importance of the attribute

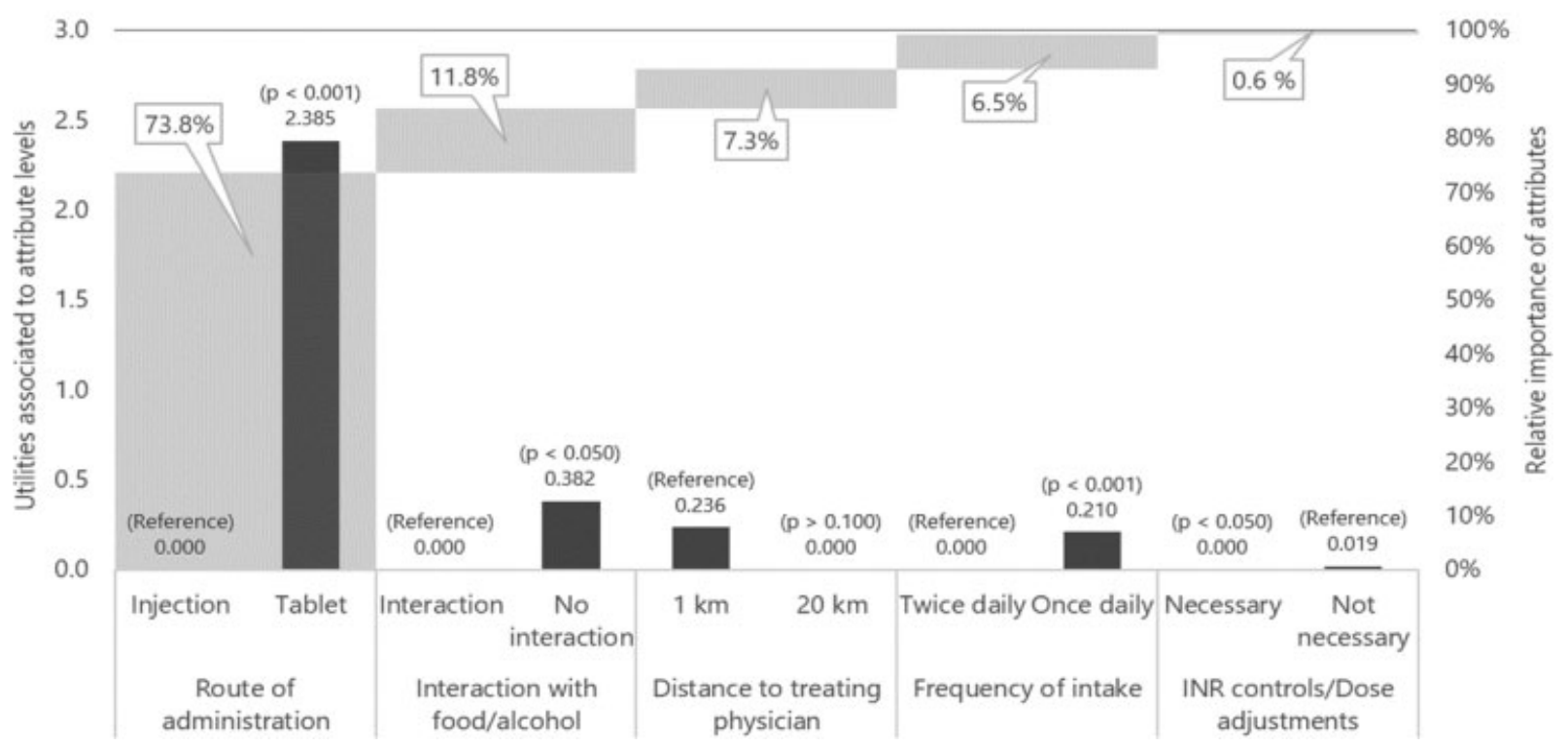

Fig. 2 Results of the conditional logit model. This figure describes the results of the conditional logit model, showing the expected utility associated with the different attribute levels and the relative importance of each attribute for a patient's decision making. INR, international normalized ratio. 


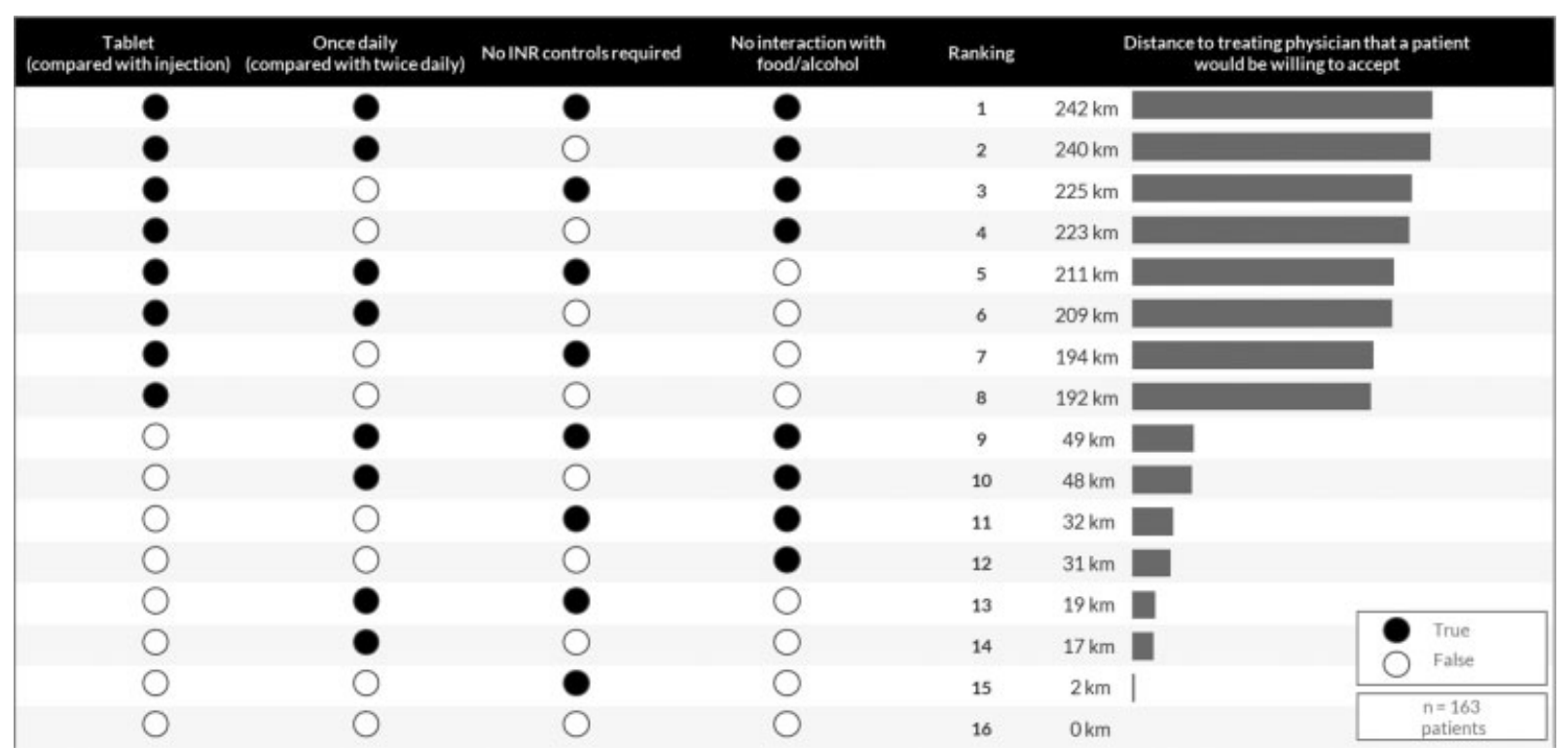

Fig. 3 Utilities for attributes expressed as distance willing to travel to treating physicians. This figure translates the calculated expected utilities associated with different combinations of attribute levels into the distance a patient would be willing to travel to the next treating physician to receive a therapy as characterized. INR, international normalized ratio.

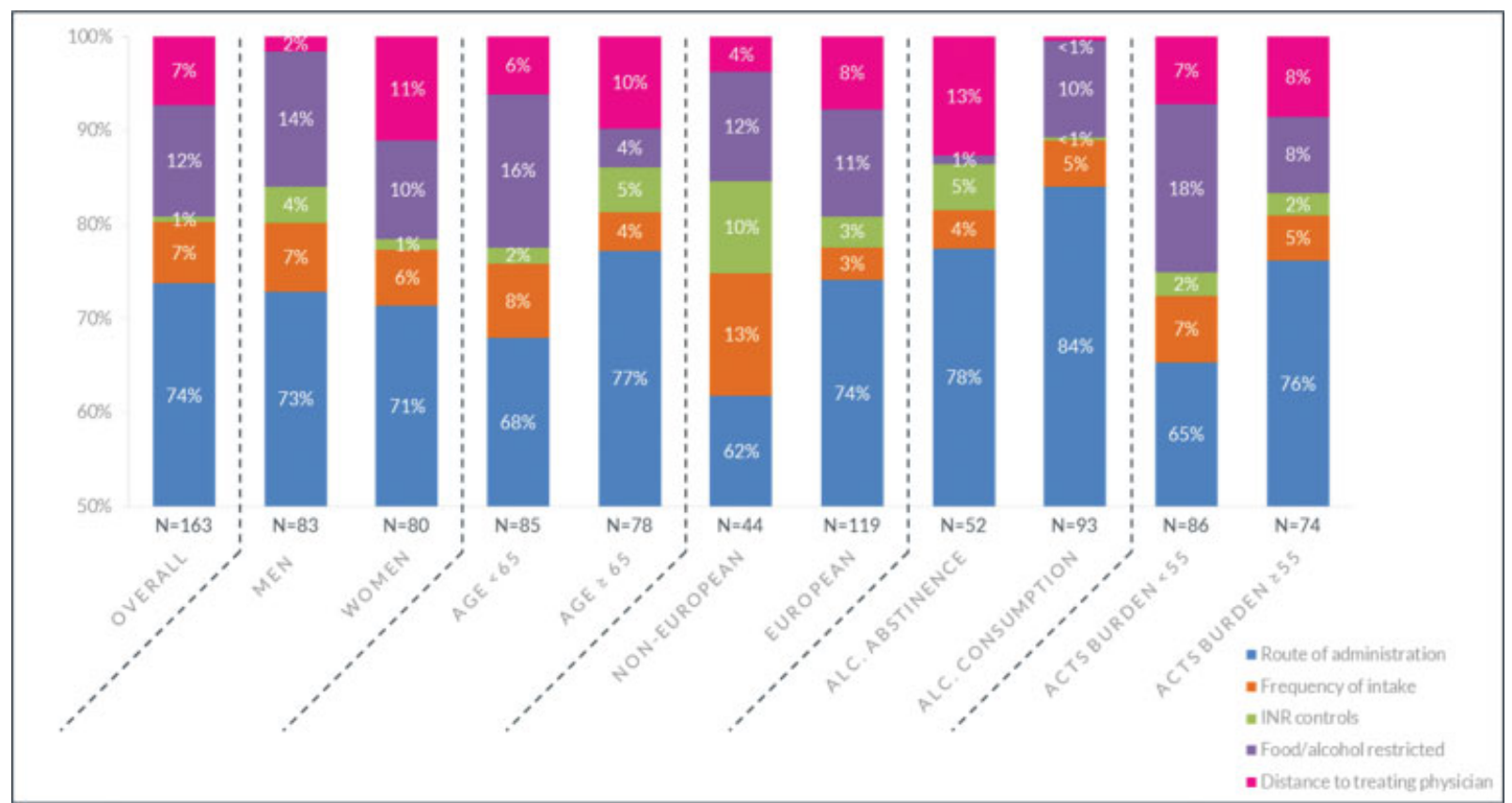

Fig. 4 Relative importance of treatment attributes stratified by different subgroups. This figure describes the relative impact of each of the five attributes stratified by patient characteristics. ACTS, Anti-Clot Treatment Scale; INR, international normalized ratio.

stronger preference for absence of restriction to food/alcohol. This attribute was also ranked higher by patients who reported a lower burden of treatment according to the ACTS (below median: 55 points) at baseline within 4 weeks of changing to DOAC, and by patients currently consuming alcohol. Furthermore, relative to the study population, patients located outside Europe (from Canada or Australia) preferred a treatment that does not require regular blood collection for INR testing, while this attribute was of minimal importance to patients located in the European continent.

\section{Discussion}

Our study provides new evidence related to the preferences of patients with cancer-associated VTE for anticoagulants. The main strength of this study is its multinational nature and the applied DCE design, which is considered as the gold standard in preference elicitation in health care. Using this approach, we were able to express the preferences of patients with cancer-associated VTE in an easier to understand unit; in the willingness to accept an additional distance to the treating physician. 
The importance of patient preference should not be underestimated and, especially in complex clinical situations such as cancer-associated VTE, the perspective of the cancer patients regarding their treatment is indispensable. In the DCE cohort, the most frequently reported reasons for the change of treatment were physician decision (35.6\%), burden of parenteral administration (25.2\%), and $\mathrm{QoL} /$ patient decision (36.8\%), which are in line with the findings of our DCE analysis, which indicated that the mode of administration was the predominant attribute for treatment-related decision making by patients. Patients placed the highest value on the convenience of an oral route of administration. Furthermore, our study clearly demonstrated that younger patients (below the age of 65 years), as well as patients who reported a lower treatment burden (ACTS score below the median of 55), rated less restrictive anticoagulants with respect to food/alcohol consumption as of higher importance. In addition, regional differences were found for non-European patients with cancer-associated VTE (mostly from Canada) who favored a lower frequency of intake substantially more than patients from Europe. Therefore, based on these results clinicians might take these patient-specific or regionally dependent preferences into consideration, when determining an optimized anticoagulation therapy in clinical practice.

To the knowledge of the authors, only a few studies have analyzed the preferences of patients with cancer-associated VTE using a choice-based methodology. ${ }^{6}$ In one study, which was also based on a DCE methodology, data from 100 patients with cancer-associated VTE treated either in Germany or the United Kingdom were presented. In that study, the mean age of patients (57 years) was significantly younger than that in our COSIMO-DCE cohort. Additionally, the risk of bleeding (major and minor), thrombosis recurrence rate, interference with cancer treatment, frequency of intake, monitoring through blood tests, and route of administration were examined in that study. The results suggested that patients most valued an anticoagulant with minimal interference with their cancer treatment (39\%), a low thrombosis recurrence rate (24\%), and a low risk of major bleeds (19\%), while preference for oral administration over injection had only moderate importance (13\%). However, these results are not directly comparable with our findings because of the differences in the DCE design, mainly in terms of selected attributes and included patients. The results from Noble et al (2015) relate to the time period before the widespread use of DOACs and are limited to the views of patients from two countries, emphasizing the need for new patient preference data in a larger and more representative population that take better account of the new therapeutic options. Our study primarily focused on the convenience attributes of the anticoagulation treatment and provides additional insights into the assessment of such treatment properties by patients with cancer-associated VTE, based on a more geographically varied patient population derived from 10 different countries. Although treating physicians in clinical practice are often able to evaluate clinical characteristics of different treatment options, our study presents insight into more unknown elements of a treatment decision, which in itself is important, as patient preferences are associated with treatment adherence/persistence and long-term therapy outcomes.

We acknowledge some limitations of our study. First, selection bias arising from differences in patient characteristics between those willing to participate in the DCE (32\%) and those who declined to participate cannot be ruled out. In principal, all observed patients in the COSIMO study $(n=505)$ were offered the opportunity to participate in the DCE by the respective study sites. Second, only those patients who changed from traditional anticoagulation with either VKA or LMWH to rivaroxaban were included. Consequently, we cannot make any statement from this study on treatment preferences of patients with cancerassociated VTE who are newly starting anticoagulation treatment or switching to other DOACs. Although generally seen as a class of drugs, DOACs differ in regimen (once vs. twice daily), in the criteria for dose reduction, and in their profile for drug-drug interactions. Therefore, it is unknown if our results are generalizable to other DOACs. Additionally, we are unable to quantify to what extent our observations were impacted by other factors in addition to the use of rivaroxaban. Third, the information presented to patients in the DCE is a simplification of reality and it is most likely that unobserved attributes (for example, drug interference, bleeding risk, or efficacy) influence the decisions of patients. Fourth, a DCE can be perceived in principle as a complex approach for both interviewers and patients, so a lack of understanding of the treatment options might have been an issue in this experiment. However, the interviewers in our study were well trained and supported by a guideline on how to design a hypothetical atmosphere within the experiment. Furthermore, the choice situations were graphically visualized and made available to patients before the interview was conducted. We excluded all patients with inconsistent responses from the final analysis by assuming that inconsistent responses are strongly correlated with a lack of understanding of the treatment choices. Finally, we did not include efficacy and safety attributes of various anticoagulant options. These relative effects could potentially influence patient preference and override any desire for convenience or lessen other burdens. However, efficacy and safety of DOACs for the treatment of cancer-associated VTE have been established in several randomized clinical trials including the Hokusai-VTE-Cancer, SELECT-D, and Caravaggio studies. ${ }^{15-17}$ Our study aimed to specifically examine convenience attributes, and therefore, in the interest of a clear focus on evaluating the convenience attributes of available therapies, our DCE assumes equal efficacy and safety for all anticoagulative therapies.

\section{Conclusion}

This study demonstrated that patients with cancer-associated VTE who changed from traditional anticoagulants to a 
DOAC primarily prefer to take an orally administered anticoagulant.

Individual preferences should be considered for the initiation and long-term treatment of patients with cancerassociated VTE as this may result in improved treatment adherence and consequently better effectiveness and safety in routine clinical practice.

\section{What is known about this topic?}

- Previous patient preference studies demonstrated that patients prefer an anticoagulant that does not interfere with their cancer treatment.

- Patients also favor efficacy and safety over convenience of drug administration.

\section{What does this paper add?}

- Our discrete-choice experiment clarifies patient preferences, especially with regard to different convenience attributes while assuming the same efficacy and safety of anticoagulation therapies.

- Decision making between convenience attributes is mainly determined by the "route of administration," indicating a strong preference for oral intake over selfinjection.

- The analysis presented provides further evidence based on a broad dataset including patients from 10 different nations.

\section{Authors' Contributions}

The authors are solely responsible for the design and conduct of this study: all study analyses, the drafting and editing of the manuscript, and its final contents. All authors read and approved the final manuscript.

\section{Funding}

This study was funded by Bayer AG. This work has been supported by the Italian Ministry of Health Ricerca Corrente - IRCCS MultiMedica.

\section{Conflict of Interest}

N.P. is an employee of Ingress-Health, who conducted the DCE on behalf of Bayer. A.Y.L. reports personal fees from Bayer, LEO Pharma, and Pfizer, and grants from BristolMyers Squibb, during the conduct of the study. A.T.C. reports grants and personal fees from Bayer during the conduct of the study; grants and personal fees from Bristol-Myers Squibb, Daiichi Sankyo Europe, and Pfizer, and personal fees from Boehringer Ingelheim, Janssen, Johnson \& Johnson, ONO Pharmaceuticals, Portola, Sanofi, and X01, outside the submitted work. A.M. reports personal fees and nonfinancial support from Bayer, during the conduct of the study; personal fees and nonfinancial support from Bayer; grants from Bristol-Myers Squibb, other from Pfizer, outside the submitted work. J.B.-W. reports grants and personal fees from Bayer, Boehringer Ingelheim, Daiichi Sankyo, Doasense, Portola, and Pfizer, outside the submitted work. L.G.M. reports personal fees from Bayer, during the conduct of the study; grants and personal fees from Boehringer Ingelheim, personal fees from Pfizer, grants from Daiichi Sankyo, outside the submitted work. K.A., S.F., M.B., and I.-M.T.-W. are employees of Bayer AG. T.W. has received honoraria from several pharmaceutical/consultancy companies (Novo Nordisk, AbbVie, Merck, GSK, BMS, LEO Pharma, AstraZeneca, Bayer, BI, and Pharmerit).

\section{Acknowledgment}

We thank all patients who participated in the COSIMO study. We thank Yoriko De Sanctis for overall statistical input in the COSIMO study.

\section{References}

1 Kearon C, Akl EA, Ornelas J, et al. Antithrombotic therapy for VTE disease: CHEST Guideline and Expert Panel Report. Chest 2016; 149(02):315-352

2 Prins MH, Bamber L, Cano SJ, et al. Patient-reported treatment satisfaction with oral rivaroxaban versus standard therapy in the treatment of pulmonary embolism; results from the EINSTEIN PE trial. Thromb Res 2015;135(02):281-288

3 Böttger B, Thate-Waschke I-M, Bauersachs R, Kohlmann T, Wilke T. Preferences for anticoagulation therapy in atrial fibrillation: the patients' view. J Thromb Thrombolysis 2015;40(04):406-415

4 Streiff MB, Bockenstedt PL, Cataland SR, et al; National comprehensive cancer network. Venous thromboembolic disease. J Natl Compr Canc Netw 2013;11(11):1402-1429

5 Dt. Gesellschaft für Angiologie - Gesellschaft für Gefäßmedizin. Diagnostik und Therapie der Venenthrombose und der Lungenembolie: AWMF Leitlinie 2015. Available at: https://www. awmf.org/uploads/tx_szleitlinien/065-0021_S2k_VTE_2016-01. pdf. Accessed July 9, 2020

6 Noble S, Matzdorff A, Maraveyas A, Holm MV, Pisa G. Assessing patients' anticoagulation preferences for the treatment of cancerassociated thrombosis using conjoint methodology. Haematologica 2015;100(11):1486-1492

7 Hutchinson A, Rees S, Young A, Maraveyas A, Date K, Johnson MJ. Oral anticoagulation is preferable to injected, but only if it is safe and effective: an interview study of patient and carer experience of oral and injected anticoagulant therapy for cancer-associated thrombosis in the select-d trial. Palliat Med 2019;33(05):510-517

8 Cohen AT, Maraveyas A, Beyer-Westendorf J, Lee AYY, Mantovani LG, Bach M; COSIMO Investigators. COSIMO - patients with active cancer changing to rivaroxaban for the treatment and prevention of recurrent venous thromboembolism: a non-interventional study. Thromb J 2018;16:21

9 Clark MD, Determann D, Petrou S, Moro D, de Bekker-Grob EW. Discrete choice experiments in health economics: a review of the literature. Pharmacoeconomics 2014;32(09):883-902

10 de Bekker-Grob EW, Donkers B, Jonker MF, Stolk EA. Sample size requirements for discrete-choice experiments in healthcare: a practical guide. Patient 2015;8(05):373-384

11 Hauber AB, González JM, Groothuis-Oudshoorn CGM, et al. Statistical methods for the analysis of discrete choice experiments: a report of the ISPOR Conjoint Analysis Good Research Practices Task Force. Value Health 2016;19(04):300-315

12 Louviere JJ, Flynn TN, Carson RT. Discrete choice experiments are not conjoint analysis. J Choice Model 2010;3:57-72 
13 Louviere JJ, Hensher DA, Swait J, Adamowicz WL. Stated Choice Methods: Analysis and Applications. Cambridge: Cambridge University Press;2010

14 Englev E, Petersen KP. ICH-GCP Guideline: kvalitetssikring af kliniske laegemiddelfors $ø$ g. Status og perspektiver. Ugeskr Laeger 2003;165(16):1659-1662

15 Raskob GE, van Es N, Verhamme P, et al; Hokusai VTE Cancer Investigators. Edoxaban for the treatment of cancer-associated venous thromboembolism. N Engl J Med 2018;378(07):615-624
16 Young AM, Marshall A, Thirlwall J, et al. Comparison of an oral factor Xa inhibitor with low molecular weight heparin in patients with cancer with venous thromboembolism: results of a randomized trial (SELECT-D). J Clin Oncol 2018;36(20): 2017-2023

17 Agnelli G, Becattini C, Meyer G, et al; Caravaggio Investigators. Apixaban for the treatment of venous thromboembolism associated with cancer. N Engl J Med 2020;382(17): 1599-1607 